\title{
Investigation of pedestrian evacuation scenarios through congestion level and crowd danger
}

\author{
Claudio Feliciani ${ }^{1}$, Katsuhiro Nishinari ${ }^{1,2}$ \\ ${ }^{1}$ Research Center for Advanced Science and Technology, The University of Tokyo \\ 4-6-1 Komaba, Meguro-ku, Tokyo 153-8904, Japan \\ feliciani@jamology.rcast.u-tokyo.ac.jp \\ ${ }^{2}$ Department of Aeronautics and Astronautics, Graduate School of Engineering, The University of Tokyo \\ 7-3-1 Hongo, Bunkyo-ku, Tokyo 113-8656, Japan
}

\begin{abstract}
In this paper, we present two quantities aimed at numerically describing the level of congestion and the intrinsic risk of pedestrian crowds. The congestion level allows to assess the smoothness of pedestrian streams and recognize regions where self-organization is difficult or not possible. This measure differs from previous attempts to quantify congestion in pedestrian crowds by employing velocities as vector entities (thus not only focusing on the absolute value). The crowd danger contains elements related to congestion, but also includes the effect of density, consequently allowing to asses the risks intrinsically created by the dynamics of crowds. Details on the computational methods related to both quantities are described in the paper and their properties are discussed. As a practical application, both measures are used to investigate supervised experiments where evacuation (or similar conditions) are considered. Results for small room sizes and limited number of pedestrians show that the crowd danger distribution over the space in front of the exit door has similar patterns to typical quantities used in the frame of pedestrian dynamics (density and flow) and symmetrical shapes are obtained. However, when larger scenarios are considered, then congestion map and crowd danger become unrelated from density and/or flow, showing that both quantities express different aspects of pedestrian motion.
\end{abstract}

Keywords: evacuation, congestion, intrinsic crowd risk, collective motion, experimental investigation

\section{Introduction}

Evacuations are one of the most common scenario studied in the frame of pedestrian dynamics and also one of the most critical situation involving pedestrians. Although evacuations represent a relatively simple case in terms of geometry, there are still many unknown aspects which need further investigations.

In the past, a number of experiments and field studies have been performed to analyse the behaviour of people during evacuations. Both humans [1-3] and animals [4,5] have been employed in experiments and granular matter [6] has been also taken as a comparative case to better understand human behaviour. Simulation models have also reached a considerable level of maturity in reproducing dynamics observed in reality (possibly due to the existence of a single common destination which makes calculations easier), thus allowing to study simple correlations between experimental conditions and evacuation time [7].

However, although several aspects have been investigated so far (influence of door width and exit location, behaviour of groups, role of leaders...) most of the studies only used the average evacuation time as a measure for effectiveness. As some researchers already pointed out, the evacuation time may not be appropriate to describe evacuation efficiency and related risks, especially considering that troubles may appear if a clog develops at the door leading to critical situations where casualties are caused by asphyxiation related to pressure waves from multiple directions $[5,8]$.

More in general, assessments in pedestrian dynamics are still based on simple quantities such as velocity and density (flow is also used which is usually defined as the product of both). While both are important and help understanding several properties of pedestrian motion, they do not allow a complete and throughout analysis since velocity is typically used as a scalar quantity and density is static by definition. For instance, a dense crowd moving in the same direction may be treated in the same way as chaotic motion 
caused, for example, by a perceived danger. If the area considered and the number of people involved are the same, then density is equal by definition, thus not accounting for the differences in motion. On the other side, when velocity is employed, then also directionality should be considered, but no agreement has been found on which method/equation should be used on this purpose.

Over the years there have been several quantities which have been proposed to describe particular properties of pedestrian motion. In particular, the bidirectional flow has attracted a lot of attention because of its capacity to form organized lanes and its similarity with physical systems. The band index [9] and the order parameter [10] have been proposed to assess the degree of stratification between different lanes, thus allowing to measure the capacity of crowds to organize themselves in efficient structures. While both are useful for analysing bidirectional streams, they are not applicable to more complex streams which are very often found in urban environments.

Alternative definitions to the classical vision of density have been also considered (a complete review is given in [11]). Among them, the Voronoi diagram has been often employed to allow defining the local density with better accuracy [12] and has become a common practice to analyse pedestrian crowds. While there are no doubts that definitions for crowdedness more proper to humans are needed, most of the proposed definitions are static by definition, therefore limiting the scope of application.

In this regard, to some extent, the work by Helbing et al. [8] has been an inspiration in developing a new quantity for pedestrian crowds. After studying the motion of pilgrims during an accident at the Hajj in Makkah, the authors came to the conclusion that so-called crowd turbulence was the leading cause to death. To assess the intrinsic risk of crowds (i.e. the amount of risk created by the crowd itself) they came up with a measure which they called "crowd pressure". By using this quantity, the researchers were able to link the moment and the location where casualties occurred with high levels of crowd pressure, thus showing that it allows to account for the dangerous effects of shock waves (which were observed and studied in detail and were found being a primary cause of injuries).

In this work, we will introduce two new quantities which are particularly useful to better understand collective behaviour during evacuations: the "congestion level" and the "crowd danger". The congestion level allows to measure the degree of organization in any kind of pedestrian flow (from unidirectional to complete random motion), while the crowd danger (also labelled as intrinsic crowd risk) helps assessing the intrinsic criticality of pedestrian crowds. Both quantities will be compared with traditional approaches using density and flow by considering controlled experiments from different authors.

\section{Congestion level and crowd danger definition}

Calculation of the congestion level makes use of velocity in the vector form and therefore, as a first step, it is necessary to obtain a vector field describing the motion of the crowd in any given moment. Two important quantities consequently need to be defined to allow the generation of the velocity vector field: grid (mesh) size and sampling time interval.

Grid size is simply the dimension assigned to each cell which will contain the velocity vector representing the average speed and direction in any location of the investigated surface. In continuous fluids, size of the mesh is not so important (or less relevant) since it is possible to measure the speed in any point. However, pedestrian crowds are more closely related to granular matter and size of the mesh need to be determined based on the particles' dimensions.

In the case of pedestrians, velocity vectors are typically extracted from trajectories (as shown in the left side of Figure 1) and therefore the portion considered also plays an important role. When very long time intervals are considered trajectories from multiple pedestrians will overlap, thus making the description of the local motion not representative of the observed dynamics.

To allow an accurate description of crowd motion with a sufficient spatial and temporal resolution, but yet capture dynamics at different densities, the combination of both mesh size and sampling time interval is fundamental. Also considering the typical size of human body and the typical range of walking speed, we concluded that a mesh size of $0.2 \mathrm{~m}$ and sampling time interval of $2.5 \mathrm{~s}$ is the most appropriate set of parameters (details of the selection are given in [3]). 
Using the above settings, a grid is generated following the steps provided in Figure 1 (on the left side). The portion of trajectory relative to the considered time interval is extracted and local velocity vectors are obtained by differentiation. Finally, an average value is obtained considering all the vectors contained in a single cell.

After obtaining the velocity vector field, the rotational (curl) is applied on the whole surface (a central differences scheme is applied) resulting in vector quantities $\vec{\omega}=[0,0, \omega]$ where only the direction perpendicular to the plane takes non-zero values (hence the first two dimensions are given as 0 ). Those nonzero values represent the vorticity, which already provides a first-instance estimation of the smoothness of motion. However, the curl returns both positive and negative values depending on the sense of rotation and high values are not necessarily synonym of high congestion. For instance, a couple dancing may return high values of rotation beside the very organized movement.

To avoid the above issues a Region of Interest (ROI) such as the one shown on the right side of Figure 1 is defined to allow a local estimation of motion dynamics. With $\omega$ as the only non-zero value of vorticity in the perpendicular direction, the level of congestion in a single ROI is defined as:

$$
C l=\frac{\omega_{\max }-\omega_{\min }}{|\vec{v}|}
$$

with $\omega_{\max }$ and $\omega_{\min }$ being respectively the maximum and minimum values of vorticity and $|\vec{v}|$ being the average absolute velocity; all relative to the considered ROI. As for the ROI dimension we found that a circular-like shape with 7 cells $(1.4 \mathrm{~m}$ ) in diameter (see right side of Figure 1) allows to describe with sufficient accuracy local changes in congestion.
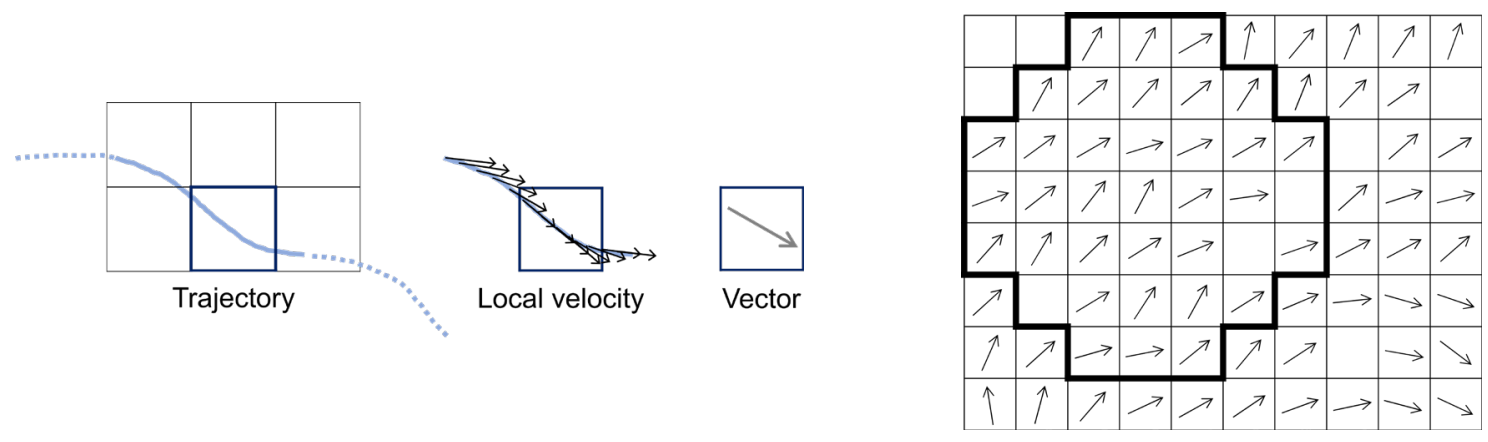

Fig. 1: Steps required to obtain a velocity vector field of moving crowds (left) and a typical vector field showing the computational ROI with thick black lines (right).

The above definition of the congestion level allows to locally measure the smoothness of motion taking into account the self-organization into groups moving in similar directions and the slow down caused by high densities. Also, since the difference between maximum and minimum values of vorticity is used, only positive values are obtained. However, while the congestion level may allow to indirectly consider the effects of density (thorough a reduction in velocity), it is not explicitly accounted for.

We may therefore define the crowd danger as the local product (considering the ROI again) of the congestion level with density $\rho$ as given by:

$$
C d=C l \cdot \rho
$$

This measure allows to take into account both the collective motion (and the corresponding degree of organization) and the pressure spread in a crowd due to its density. Considering that both the amount of crowdedness and the way people are moving are accounted for, it can be a measure of the intrinsic crowd risk and hence the name crowd danger. 


\section{Calibration using controlled experiments}

To understand the properties of congestion level and crowd danger several characteristic experiments from multiple sources have been analyzed (details are given in [3]). In particular, three types of experiment were considered for comparison: unidirectional, bidirectional and chaotic (multidirectional) motion. Those cases were chosen because of their scientific significance and the availability of experimental data.

The three cases are schematically depicted in Figure 2 using different colors for each class of pedestrians moving in a particular direction. In unidirectional streams, people all move in the same direction and, although overtaking is still possible, a very smooth motion is usually observed. In the case of bidirectional streams interactions become stronger, but lanes are typically formed (if possible) to reduce collisions with the counter-flow. Finally, multidirectional streams are usually chaotic, especially if densities are high, since trajectories often intersect with other pedestrians.
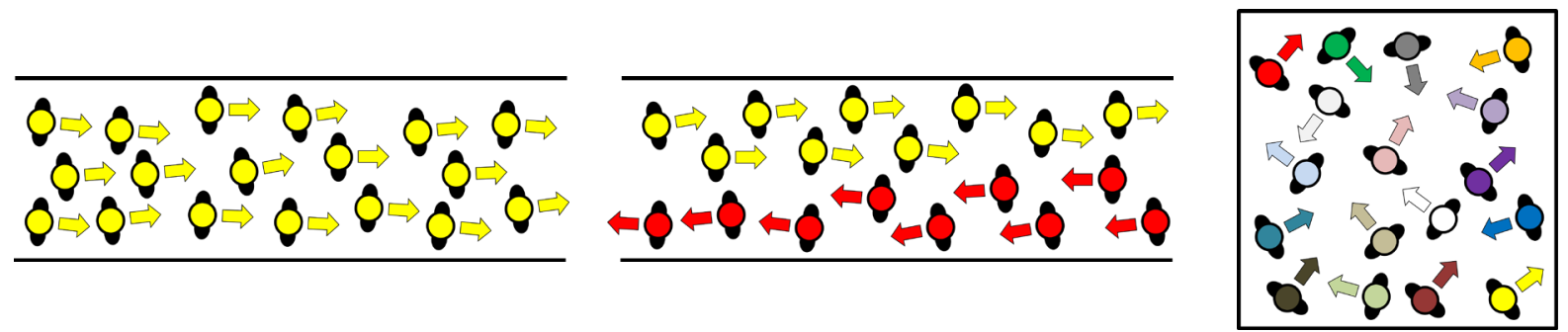

Fig. 2: Different types of pedestrian streams: unidirectional, bidirectional and chaotic (multidirectional) motion (from left to right). Colours are used to distinguish each group of pedestrians based on their destination.

Literature data have been used for the unidirectional and bidirectional case (considered studies are described in [13] and data are available in [14]), while own experiments were performed for the chaotic case (details are given in $[3,15,16])$. For the latter, two different configurations were tested: in one case participants were simply asked to move inside a room (free chaotic motion) while in a second case some additional people were asked to traverse it in different directions (forced chaotic motion), thus making the formation of organized structure more difficult (and almost impossible).
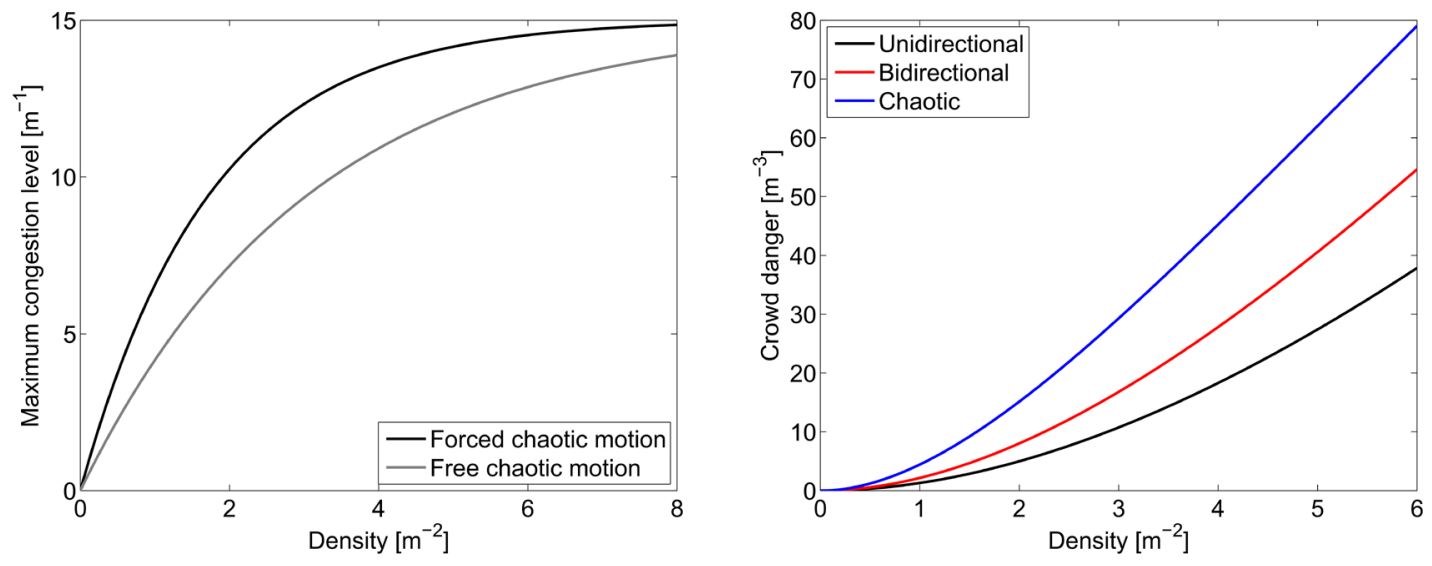

Fig. 3: Maximum congestion level measured at different densities in the chaotic experiment for different configurations (left). Crowd danger evolution for different types of pedestrian streams (right). In all the cases numerical values are derived from experiments (with details provided in [3]). Chaotic curve in the right graph also considers additional chaotic experimental conditions not accounted for in the left graph.

Figure 3 (left) shows the congestion level measured at different densities for the two chaotic experiments described earlier. Results showed that there is a typical maximum value of congestion level 
associated with a particular density and experimental configuration. Also, we noticed that the maximum congestion level converged toward a plateau of $15 \mathrm{~m}^{-1}$. While experimental evidence may not be sufficient to come at final conclusions, it may be possible that this value represents a property of pedestrian crowds, in a sort of cognitive process which reduce speed to limit the consequences of collisions which are getting more frequent at higher densities.

When typical values of the crowd danger were computed for different types of flow, we noticed that they all followed an exponential-like behaviour as given on the right side of Figure 3. As the graph shows, the intrinsic crowd risk grows slowly (in relation to density) when unidirectional motion is considered, because movements are synchronized among individuals. However, in case of a crowd moving in multiple directions there is a steeper increase in crowd danger, since movements are more random and collisions (or very close interactions) are frequent. While we have not been able to provide a characteristic cautionary value for crowd danger (also because of the exponential nature of the function), we speculate that above 75 $\mathrm{m}^{-3}$ attention is required as accidents are more likely.

\section{Results}

To further demonstrate the properties of the congestion level and the crowd danger, show possible applications and better study evacuation dynamics, we considered three experiments from different contexts. A representative image of each experiment is given in Figure 4 and similarities/differences among the different cases will be shortly discussed before introducing the results.

The first set of experiments (left in Figure 4, details are provided in [1]), were performed in a squared room $5 \mathrm{~m}$ in side length. In the centre of it a smaller area $3 \mathrm{~m}$ in side length (clearly visible in Figure 4) was used to gather participants at the beginning of the experiment. After the "start" was given, people rushed to the exit and left the room following a predetermined wide path (i.e. the area in front of the exit was unobstructed allowing a smooth evacuation). Exit door had a variable width of $0.8 \mathrm{~m}$ and $1.2 \mathrm{~m}$. This research particularly focused on group behaviour during evacuations and therefore groups of several sizes (from 2 to 8 members) were created among participants (interactions within the social groups also varied among each experimental run). Also, it is important to notice that participants were all children of different ages (from 10 to 12 and from 15 to 17 years old). Number of participants varied from a minimum of 22 to a maximum of 50 people. In total 51 experimental runs are considered. Beside the differences in each case, after examining the videos, we determined that for the scope of this study, the whole set of experiments can be regarded as a single scenario, especially due to the similarity of the experimental geometry.

The second set of experiments (centre in Figure 4, details provided in [3]) consisted in a room of $4 \mathrm{~m}$ in width and $7 \mathrm{~m}$ in length, with a $0.8 \mathrm{~m}$ exit located in the middle of the longest side. Participants (university students in their 20s) gathered concentrically around the exit before the evacuation order was given. A variable number of participants was considered, with 3 runs having 25 participants and 5 runs with 43 participants. All people behaved individually. Also in this case, we deemed appropriate to consider the 8 experimental runs altogether since geometry had not been varied.

The last experiment considered (right in Figure 4, details provided in [17]) consists in a large-scale reproduction of the entrance of a music event. While in this case motivation was not for leaving the room, but rather for entering it, it still represents an interesting case for a bottleneck geometry. 273 young people aged between 20 and 30 gathered for the experiment. In contrast to the previous cases, two small paths allowing only one person each were made available for "evacuation" (both are visible in Figure 4). For this experiment only one execution was performed and participants were specifically asked to imagine that their idol is present at the concert, thus having them trying to enter quickly to get the best places. As a result, pushing and forced motion were frequently seen in different moments of the experiment.

For each experimental run, several heat maps for density, flow, congestion level and crowd danger were computed and are presented in Figure 4. To increase the accuracy for the maps representing the congestion level (and consequently crowd danger) the $2.5 \mathrm{~s}$ sampling interval discussed earlier was used, but it has been moved in $0.1 \mathrm{~s}$ steps, thus generating a large number of maps also for short experimental runs. In all the cases, densities have been computed by using the Voronoi method [12] and flow was obtained by multiplying local speed (taken over 5 frames) with corresponding density. Mesh size is of 0.2 
$\mathrm{m}$ as a result of the previous discussion on the computational method of the congestion level. Each experimental run is considered from the moment where the first person exits the room until the last one pass through the exit. Finally, a generic filter has been used to remove outliers resulting from tracking inaccuracies (difference with unfiltered results was judged minimal, thus allowing its use).
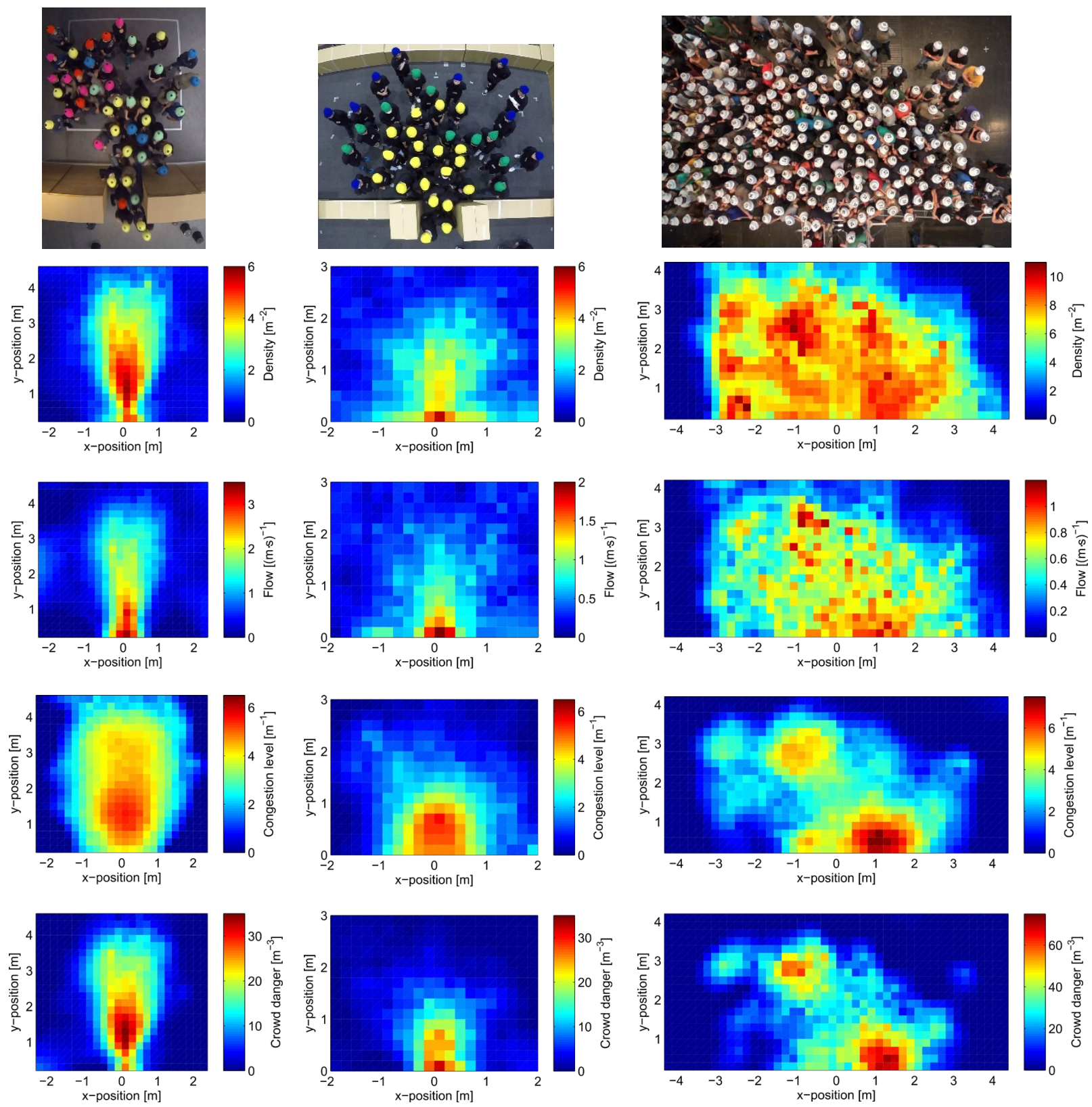

Fig. 4: Heat maps for different quantities (density, flow, congestion level and crowd danger) for the three experimental scenarios considered. In all the cases, the exit (or the centre of it) is located at the origin $(0,0)$. Figures are cropped to focus on the region in front of the exit (complete experimental area is bigger).

Results from Figure 4 show quite different patterns depending on the quantity considered and the experiment being investigated.

In the experiment involving children provided on the left, it is seen that from a qualitative point of view the 4 considered quantities are similar. Density, congestion level and crowd danger all show 
their maximum in a location about $1-1.5 \mathrm{~m}$ in front of the exit. Only the flow shows a quite different shape by peaking right in the location of the exit. In particular, a very close similarity is found between the density map and the one for the crowd danger, which could be partially explained considering that density is involved in the calculation of the latter. The congestion level shows the largest distribution, with comparatively high values of congestion also found where density and flow are low. In interpreting those results one should be reminded that children gathered in the centre of the room before the experiment was started and this could partially explain the high values found in inner parts of the room.

When the second set of experiments is considered, whose results are given in the central part of Figure 4 , then a partially different picture is obtained considering evacuation dynamics. While the congestion level still shows a large dispersion roughly centred in a location around $0.5-1 \mathrm{~m}$ in front of the exit, all the other quantities show their maximum right in the place of the exit. This could be partially associated with the method employed to compute the Voronoi cells, which, although it partially considers the opening created from the door, may still overrepresent the density right in front of the exit. Nonetheless, both experiments involving children (left) and young adults (centre) employs the same method in computing Voronoi density, so this could not be the only cause explaining the high values observed at the exit. An interesting observation can be done by comparing the flow for the case with children (left) with the one for adults (centre). The maximum flow for children is considerably higher than typical figures from the literature [18] (usually in the range of $2-2.5$ pedestrians $\left.\left(\mathrm{m}^{\cdot} \mathrm{s}\right)^{-1}\right)$. While this could be explained with partially different definitions for flow, the smaller body size of children is a more possible reason.

Finally, the large experiment relative to the concert entrance, whose results are given on the right of Figure 4, is discussed. In this case, a very different image is obtained among the different quantities and also compared to the previous cases. While high density values are found throughout the whole experimental area, congestion level and crowd danger both have two peaks found close to the right "exit" and in a region lying far from the egress location. The different distributions found show that congestion level has a different nature compared to density and flow and it possibly allow to determine the selforganization of motion for pedestrian crowds. When the crowd danger is considered, then higher values (more than doubles) are obtained compared to the previous case. This is a combination of the higher densities and higher values of congestion and it qualitatively reproduce well the general impression obtained by visioning the video of the experiment.

As a final remark, it is seen that in most of the cases the most congested region lied at a distance similar to the width of the exit door right in front of the exit. Previous studies (involving granular matter, humans and sheep) already found that the flow is increased by placing an obstacle in this region $[2,5,6]$, which may cause an overall reduction in congestion.

\section{Conclusion}

In this study, evacuation experiments for different types of pedestrian crowds have been studied employing typical quantities usually associated with pedestrian traffic (density and flow) and newly defined measures to assess the level of congestion and the intrinsic risk created by the motion of the crowd.

Results showed that while there are common characteristics among the scenarios involving a small number of participants in clearly defined and symmetric contexts, all defining a congested region right in front of the exit, the situation changed when a more realistic case was studied.

This shows that while it is possible to prevent accident by focusing on hotspots which are generally considered as dangerous, in reality each situation needs to be considered separately and crowd monitoring and management are important aspects to guarantee safe and smooth mass events.

The methods presented in this work may found applications in several contexts involving pedestrian traffic, with evacuation being only an example. In particular, the congestion level and the related crowd danger can be useful for designing pedestrian facilities since dangerous and congested regions are easily recognized. In addition, a use in conjunction with simulation models may also help analysing the results and identify locations which need improvements.

Although both quantities work best on large database of similar experiments (as presented in this study), it may be also possible to employ them for real-time crowd analysis, for example during mass events, 
when simple but yet meaningful information are important to highlight location which need a prompt intervention by security personnel.

\section{Acknowledgements}

This work was financially supported by JST-Mirai Program Grant Number JPMJMI17D4, the Doctoral Student Special Incentives Program (SEUT RA) and the Foundation for Supporting International Students of the University of Tokyo. In addition, we are grateful to the researchers who openly shared their data.

\section{References}

[1] C. von Krüchten, F. Müller, A. Svachiy, O. Wohak, A. Schadschneider, "Empirical study of the influence of social groups in evacuation scenarios", in Traffic and Granular Flow '15, L. V. Knoop, Springer International Publishing, 2016, pp. 65-72.

[2] D. Yanagisawa, A. Kimura, A. Tomoeda, R. Nishi, Y. Suma, K. Ohtsuka, K. Nishinari, "Introduction of frictional and turning function for pedestrian outflow with an obstacle", Phys. Rev. $E$, vol. 80, no. 3, 2009.

[3] C. Feliciani, K. Nishinari, "Measurement of congestion and intrinsic risk in pedestrian crowds", Transp. Res. Part. C Emerg. Technol., vol. 91, pp. 124-155, 2018.

[4] P. Lin, J. Ma, T. Liu, T. Ran, Y. Si, T. Li, "An experimental study of the "faster-is-slower" effect using mice under panic", Physica A, vol. 452, pp. 157-166, 2016.

[5] A. Garcimartín, J. M. Pastor, L. M. Ferrer, J. J. Ramos, C. Martín-Gómez, I. Zuriguel, "Flow and clogging of a sheep herd passing through a bottleneck", Phys. Rev. E, vol. 91, no. 2, 2015.

[6] I. Zuriguel, D. Ricardo Parisi, R. C. Hidalgo, C. Lozano, A. Janda, P. A. Gago, J. P. Peralta, L. M. Ferrer, L. A. Pugnaloni, E. Clément, D. Maza, I. Pagonabarraga, A. Garcimartín, “Clogging transition of many-particle systems flowing through bottlenecks", Sci. Rep., vol. 4, 2014.

[7] L. Lu, C. Y. Chan, J. Wang, W. Wang, "A study of pedestrian group behaviors in crowd evacuation based on an extended floor field cellular automaton model", Transp. Res. Part. C Emerg. Technol., vol. 81, pp. 317-329, 2017.

[8] D. Helbing, A. Johansson, H. Z. Al-Abideen, "The Dynamics of Crowd Disasters: An Empirical Study", Phys. Rev. E, vol. 75, no. 4, 2007.

[9] K. Yamori, "Going with the flow: Micro--macro dynamics in the macrobehavioral patterns of pedestrian crowds", Psychol. Rev., vol. 105, no. 3, 1998.

[10] S. Nowak, A. Schadschneider, "Quantitative analysis of pedestrian counterflow in a cellular automaton model", Phys. Rev. E, vol. 85, no. 6, 2012.

[11] D. C. Duives, W. Daamen, S. P. Hoogendoorn, "Quantification of the level of crowdedness for pedestrian movements", Physica A, vol. 427, pp. 162-180, 2015.

[12] B. Steffen, A. Seyfried, "Methods for measuring pedestrian density, flow, speed and direction with minimal scatter", Physica A, vol. 389, no. 9, pp. 1902-1910, 2010.

[13] J. Zhang, W. Klingsch, A. Schadschneider, A. Seyfried, "Ordering in bidirectional pedestrian flows and its influence on the fundamental diagram", J. Stat. Mech., vol. 2012, no. 2, 2012.

[14] Forschungszentrum Jülich, "Data archive of experimental data from studies about pedestrian dynamics", http://ped.fz-juelich.de/db/ (accessed May, 2018).

[15] H. Yamamoto, D. Yanagisawa, C. Feliciani, K. Nishinari, "Modeling body-rotation behavior of pedestrians for collision avoidance in a narrow corridor", Transp. Res. Part. B Meth., 2018 (submitted).

[16] C. Feliciani, K. Nishinari, "Estimation of pedestrian crowds' properties using commercial tablets and smartphones", Transportmetrica B, 2017 (submitted).

[17] A. Sieben, J. Schumann, A. Seyfried, "Collective phenomena in crowds-where pedestrian dynamics need social psychology", PLoS ONE, vol. 12, no. 6, 2017.

[18] A. Seyfried, O. Passon, B. Steffen, M. Boltes, T. Rupprecht, W. Klingsch, "New insights into pedestrian flow through bottlenecks", Transp. Sci., vol. 43, no. 3, 2009. 